\title{
Affective disorders and risk of developing dementia: systematic review
}

Joaquim da Silva, Manuel Gonçalves-Pereira, Miguel Xavier

and Elizabeta B. Mukaetova-Ladinska

\section{Background}

Affective disorders are associated with cognitive disturbances but their role as risk factors for dementia is still not fully investigated.

\section{Aims \\ To evaluate the risk of developing dementia in individuals with a history of affective disorder.}

\section{Method}

We conducted a systematic review of case-control and cohort studies addressing the risk of developing dementia in people with affective disorders. To the best of our knowledge, this is the first systematic review that has included studies evaluating this risk specifically in people with bipolar disorder.

\section{Results}

Fifty-one studies were included. Most of the studies found an increased risk for developing dementia in individuals with depression. Greater frequency and severity of depressive episodes seem to increase this risk. The evidence is contradictory regarding whether there is a difference in risk in people with early- or late-onset depression. The few available risk estimates for dementia in people with bipolar disorder suggest an even higher risk than for those with depression.

\section{Conclusions}

Affective disorders appear to be associated with an increased risk of developing dementia, and one that is dependent on clinical and demographic variables. Depression may be both a prodrome and a risk factor for dementia. Future research should aim to elucidate the mechanisms that mediate these links.

\section{Declaration of interest}

None.
Dementia is a disabling clinical syndrome characterised by a progressive deterioration of cognition associated with impairment in activities of daily living. ${ }^{1}$ It is on the increase in high-income countries and even more so in low- and middle-income countries. ${ }^{2}$ At present, about 36 million people in the world live with dementia and this number will almost double in the next 20 years, thus imposing a great burden on patients, their families and society as a whole. ${ }^{3}$ Because the current dementia treatments are still limited, a broad understanding of risk factors is essential for the prevention of these diseases.

Affective disorders are associated with cognitive disturbances that are not just limited to acute mood episodes. Even after major depression has remitted, patients show impairment in executive function and attention. ${ }^{4}$ Similarly, euthymic individuals with bipolar disorder have deficits in verbal declarative memory and executive function. ${ }^{5}$ Furthermore, structural brain abnormalities are found in people with affective disorders, including hippocampal atrophy in those with a history of depression ${ }^{6,7}$ and white matter changes in individuals with bipolar or major depressive disorder, especially those with late-onset depression. ${ }^{8}$ Regarding the autopsy of a patient with bipolar disorder, Kraepelin ${ }^{9}$ wrote: 'The post-mortem showed widespread arterio-sclerosis of the blood vessels of the brain. This termination appears to be not altogether unusual in maniacal depressive insanity'. A wide gap prevailed between this observation made at the start of the twentieth century and the recent interest in affective disorders as risk factors for late-life dementia. A growing number of epidemiological studies have addressed this link, most of them focused on depression as a risk factor for Alzheimer's disease. In fact, two meta-analyses found that having a history of depression approximately doubled the probability of developing Alzheimer's disease $^{10}$ or dementia in general. ${ }^{11}$ However, in the more recent meta-analysis ${ }^{10}$ the results were calculated by pooling crude odds ratios from different studies. This may have led to some bias because important confounding factors such as age, gender and education were not generally accounted for. Furthermore, in order to calculate a pooled odds ratio from a group of homogeneous studies, strict selection criteria were used in these meta-analyses. This strategy may have excluded studies with relevant contributions to our understanding of the association between depression and dementia. Despite the aforementioned meta-analyses, this putative link is still the target of much controversy with the dispute over prodrome $v$. risk factor at the core. Byers \& Yaffe ${ }^{12}$ have recently revisited this subject. However, two issues may have limited their conclusions. They reviewed only studies published in 2000 or later, and the determinant criteria for including a study was relevance as judged by the authors.

In contrast to depression, and to the best of our knowledge, there are no published systematic reviews looking at bipolar disorder as a risk factor for dementia. We therefore conducted a thorough systematic review of longitudinal observational studies that evaluated affective disorders as potential risk factors for dementia. For this review we considered studies evaluating depression (major depression, minor depression or an increased level of depressive symptoms) and bipolar disorder (mania or hypomania). We considered dementia in general or specific subtypes of dementia (Alzheimer's disease, vascular dementia, frontotemporal dementias and dementia with Lewy bodies) as outcomes. By using appropriate search expressions and broadly defined criteria, we intended to consider a wide range of relevant studies. By doing so, we aimed to provide a more comprehensive perspective than the ones provided by previous reviews and meta-analyses.

\section{Method}

\section{Data sources}

We performed a systematic review using strategies recommended to retrieve aetiological studies (cohort and case-control studies). ${ }^{13}$ 
A first search block was constructed by using cohort studies OR risk OR [odds AND ratio*] OR [relative AND risk] OR casecontrol $^{\star}$ OR [case-control studies] as search terms. This first block was intersected with a second block created using the following keywords: [dementia OR Alzheimer disease OR vascular dementia OR Frontotemporal dementia OR Frontotemporal lobar degeneration OR Lewy body disease OR Lewy body dementia] AND [depression OR depressive OR unipolar disorder OR mania OR bipolar disorder OR manic-depress ${ }^{*}$. The correspondent Medical Subject Heading (MeSH) terms were used where available.

We used this strategy to undertake an electronic search of PubMed, PsycINFO and LILACS for references available up to June 2011. The publications found were reviewed by one of the authors (J.d.S.). The titles of identified references were reviewed and the ones highly unlikely to be relevant were disregarded. Next, the abstracts of the remaining articles were appraised. Relevant studies were read in full and their references were searched for suitable articles. Only peer-reviewed articles were considered and no language restrictions were applied. Authors were contacted by email when doubts regarding study design or presented data were hindering the decision whether to include them in the analysis.

\section{Inclusion and exclusion criteria}

Inclusion criteria were:

(a) study type: longitudinal studies either retrospective or prospective that evaluated the risk of developing dementia in people with affective disorders. Studies that compared the risk of developing dementia between different affective disorders (e.g. unipolar depression $v$. bipolar depression) were also included;

(b) participants: adults older than 18 years of age;

(c) outcome: any measure of risk (for example odds ratio, relative risk, hazard ratio) or results presented in a way that a crude odds ratio or relative risk could be calculated.

Exclusion criteria were:

(a) studies that did not describe the method for diagnosing dementia or depression;

(b) studies that did not specify the method of quantifying depressive symptoms in the cases where a continuous measure was used;

(c) longitudinal studies specifically addressing samples of people with cognitive impairment or subjective memory impairment at baseline.

\section{Study quality and data extraction}

The quality of studies was assessed using the Newcastle-Ottawa quality assessment scale. ${ }^{14}$ This instrument was developed to assess the quality of both cohort and case-control studies and it is centred on three main categories: (a) selection of groups; (b) comparability of the groups; and (c) assessment of exposure in case-control studies or outcome of interest in cohort studies (online Tables DS1 and DS2). The studies were scored by one of the authors (J.d.S.) and a sample of these was cross-rated by another author (M.G.-P.) to improve reliability.

Study details, including country where it was conducted, type of study (case-control or cohort study), dementia and depression diagnostic criteria, sample size and study outcomes, were extracted using a standardised form. If available, adjusted measures of risk were collected, together with an indication of the variables that were controlled for. Otherwise, crude measures of risk were retrieved or calculated from the results. Information regarding clinical and demographic characteristics that influenced the risk of developing dementia was also collected.

\section{Reporting study results}

Starting with the studies that focused on depression, we report the risk estimates of developing dementia with a $95 \%$ confidence interval and address clinical and demographic characteristics that seem to modulate the magnitude of this risk (gender, number of depressive episodes, severity of depression, age at depression onset and apolipoprotein E (APOE) status). Finally, we describe the few studies evaluating the risk of developing dementia in people with bipolar disorder and report their outcome risk measure with a 95\% confidence interval. Forest plots were rendered using MatLa R2010a (The MathWorks, Natick, USA, www.mathworks.com; Mac version). A custom code was used and it is available on request from the authors.

\section{Results}

The electronic search provided 4109 publications for analysis, of which a total of 51 publications met the inclusion criteria. ${ }^{15-65}$ Of these, all had information regarding depression and the risk of developing dementia, but only five studies presented data in relation to this risk in bipolar disorder ${ }^{19,26,27,38,41}$ (Fig. 1). The quality assessment of the publications included is summarised in Tables DS1 and DS2.

\section{Depression as a risk factor for late-life dementia}

Although the majority of published studies to date highlight an increased risk of late-life dementia in people with a previous diagnosis of depression, ${ }^{15,17-26,33,35,37,38,40,42,44,45,47-54,57-60,62-65}$ approximately a quarter of the studies did not show a statistical significant increase in this risk (online Tables DS3 and DS4, Figs 2-4). ${ }^{16,28,30-32,34,39,43,46,55,56,61}$ A protective effect of depression was only described in one study. ${ }^{36}$ This isolated finding might

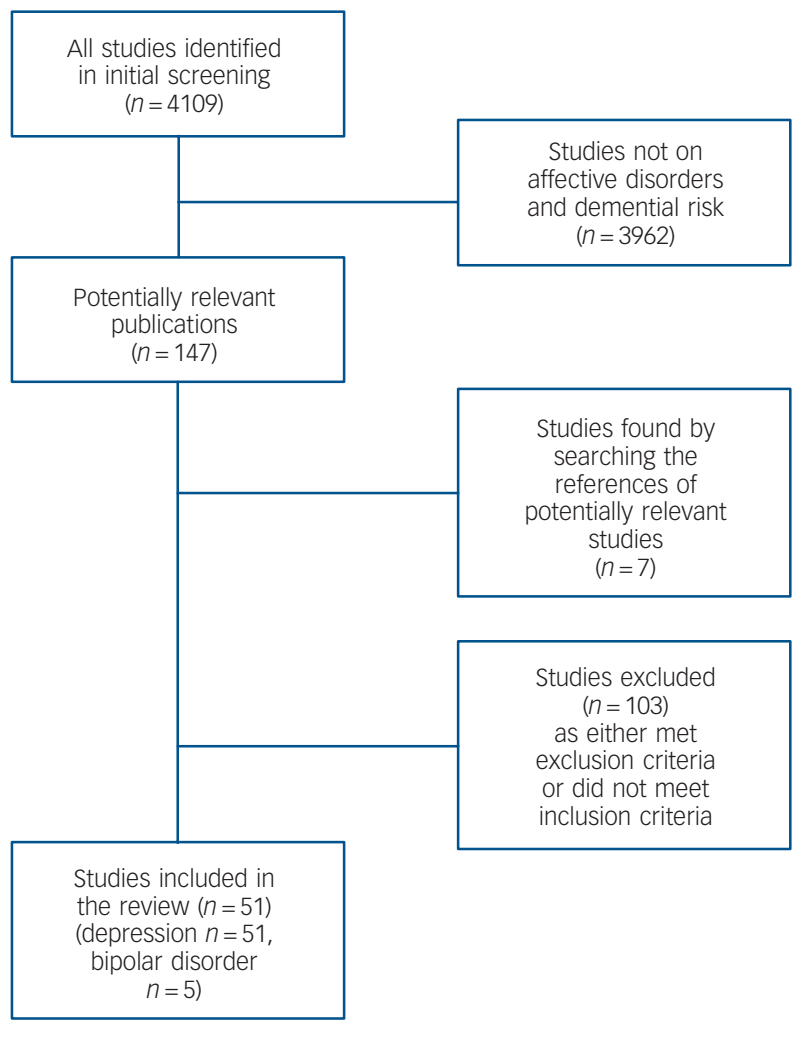

Fig. 1 Flow chart of literature search and study selection. 
be explained by the use of controls from populations with a high prevalence of depression (caregivers and elderly people living in nursing homes). ${ }^{36}$

There were only four studies providing results specifically with vascular dementia as an outcome. ${ }^{33,50,62,64}$ Two of these studies reported a statistically significant risk of developing this type of dementia in patients with depression. ${ }^{33,64}$ Lenoir et al ${ }^{64}$ found a significant risk for vascular dementia but not for Alzheimer's disease, while Irie et al ${ }^{50}$ found exactly the opposite using the same method to define depression. ${ }^{66}$ In this last study the sample was exclusively composed of men, which may have contributed to these contradictory results. Only one study reported the effect of depression on the risk of developing mixed dementia, showing an increased risk for this subtype of dementia (hazard ratio $(\mathrm{HR})=2.8,95 \%$ CI $1.5-5.1) .{ }^{50}$ No study reported depression as a risk for developing dementia with Lewy bodies or frontotemporal dementia.

\section{Risk of dementia according to the characteristics of depression: frequency, duration, severity and onset}

Greater severity of depressive symptoms at baseline was associated with a higher risk of developing dementia ${ }^{60}$ and Alzheimer's disease. $^{35,44,50,51,60}$ Moreover, a large case register-based cohort study in Denmark showed that the number of depressive episodes leading to admission increased the risk of being readmitted with a diagnosis of dementia. However, the difference was only significant when individuals with one depressive episode were compared with people who had more than four episodes $(\mathrm{HR}=6.16$, CI $95 \% 1.39-27.22) .{ }^{41}$ Similarly, in a community cohort with a long follow-up period (median 23.6 years), Dotson et $a l^{59}$ reported a dose-response relationship between the number of what they called 'episodes of elevated depressive symptoms' and the risk of developing dementia. Nonetheless, one study ${ }^{44}$ found that participant-reported duration of depression was not predictive of Alzheimer's disease $(\mathrm{OR}=1.01,95 \%$ CI $0.88-1.15)$ or dementia $(\mathrm{OR}=1.04,95 \%$ CI $0.97-1.11)$. In this study, however, it was not clear what was measured with this self-report (for example either total duration of depressive episodes, or duration of present or last episode).

Age at onset of depression (early- and late-onset depression specifiers) has also been used to explore whether depressive illness is a real aetiological risk factor for dementia, an early clinical manifestation (prodrome) or a psychological reaction to the disease process. $^{20,22,24,26,29,31,37,44,50,53,54}$ Most of the studies evaluated only late-onset depression or late-life depression. Some also looked at early- and late-onset depression or late-life depression at the same time. ${ }^{20,24,27,43,54,58}$ However, contradictory findings undermine a straightforward conclusion, with data supporting an increased risk only for late-onset depression, ${ }^{24,58}$ early-onset depression alone $e^{20,30,54}$ or both ${ }^{37}$ (Fig. 5). In the latter study, ${ }^{37}$ the authors went further and presented risk estimates depending on the interval (in years) between onset of depression and Alzheimer's disease onset. Although the risk of developing dementia was greater for people who had a first episode of depression a few years before the onset of cognitive impairment, the risk was also significantly increased for individuals with much earlier onset of depression (25 years before the onset of dementia). ${ }^{37}$ However, the study had some limitations, the most obvious being the method for diagnosing depression (Table DS3).

\section{Gender differences and risk of dementia in patients with late-life depression}

Although gender differences were generally controlled for in most of the analysed studies, few publications evaluated separately the risk for women and men. In the PAQUID study, depressive symptoms at baseline increased the risk for developing Alzheimer's disease only in men (odds ratio $(\mathrm{OR})=3.7,95 \% \mathrm{CI}$ $1.7-7.9$ for men and $\mathrm{OR}=0.6,95 \%$ CI $0.3-1.3$ for women). ${ }^{39}$ Likewise, Dal Forno et $a l^{42}$ reported an increased risk of Alzheimer's disease only in males with increased depressive symptoms $\quad(\mathrm{OR}=1.06,95 \%$ CI $1.04-1.09$ for men, and OR $=0.99,95 \%$ CI $0.95-1.02$ for women). The results were similar
Wetherell et al (1999) 28

Cooper \& Holmes (1998) $)^{26}$ Brommelhoff et al (2009) $)^{58}$ Agbayewa (1986) ${ }^{16}$

Zalsman et al (2000) ${ }^{32}$

Broe et al (1990) ${ }^{18}$ speck et al (1995) 20 Steffens et al (1997) ${ }^{24}$ Brommelhoff et al (2009) ${ }^{58}$ Kokmen et al (1991) ${ }^{19}$ Green et al (2003) ${ }^{37}$ French et al (1985) ${ }^{15}$ Shalat et al (1987) ${ }^{17}$ Tsolaki et al (1997) ${ }^{25}$ Zubenko et al (2003) ${ }^{36}$ Hébert et al (2000) ${ }^{33}$

9

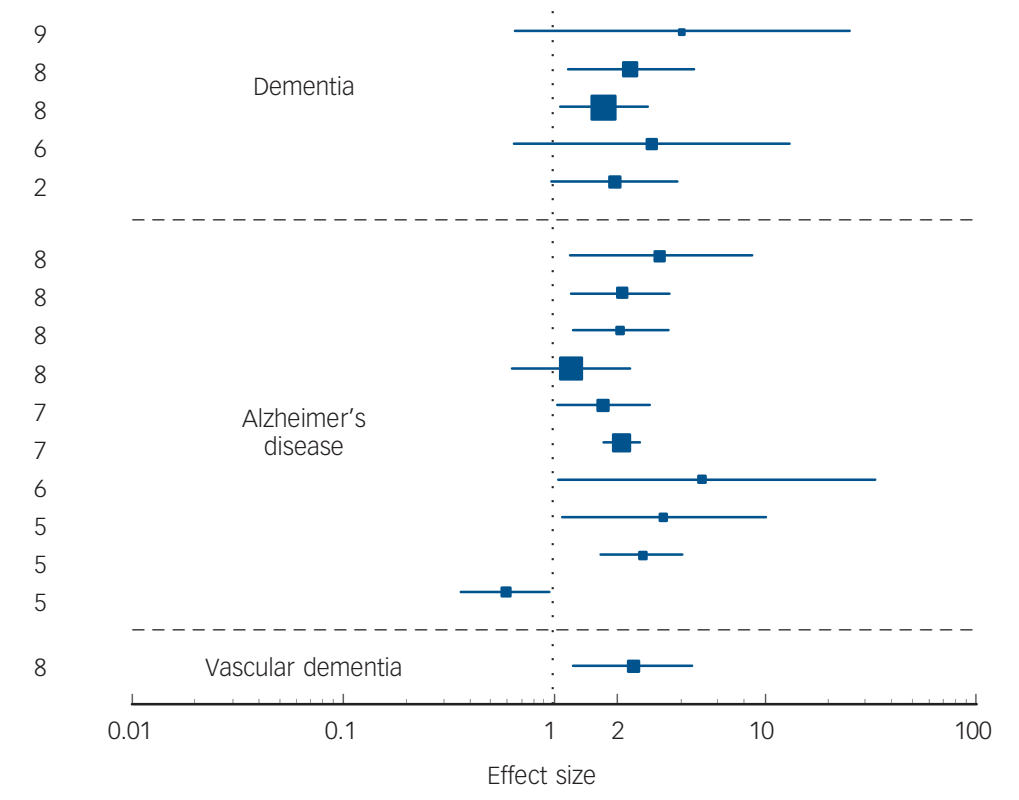

$4.00(0.64-25.16)$

$2.31(1.16-4.61)$

$1.72(1.07-2.76)$

$2.90(0.64-13.15)^{\mathrm{a}}$

$1.94(0.98-3.84)$

$3.20(1.20-8.70)$

$2.10(1.20-3.50)$

$2.08(1.22-3.52)$

$1.20(0.63-2.30)$

$1.71(1.03-2.83)$

$2.08(1.70-2.55)$

$5.00(1.04-33.00)$

$3.30(1.10-10.10)$

$2.60(1.65-4.09)^{\mathrm{a}}$

$0.59(0.36-0.95)^{a}$

$2.41(1.22-4.52)$

Fig. 2 Forest plot of case-control studies that evaluated depression as a risk factor for dementia, Alzheimer's disease or vascular dementia.

Studies are organised from high quality (top) to lower quality (bottom). OR, odds ratio. a. Crude odds ratio. 


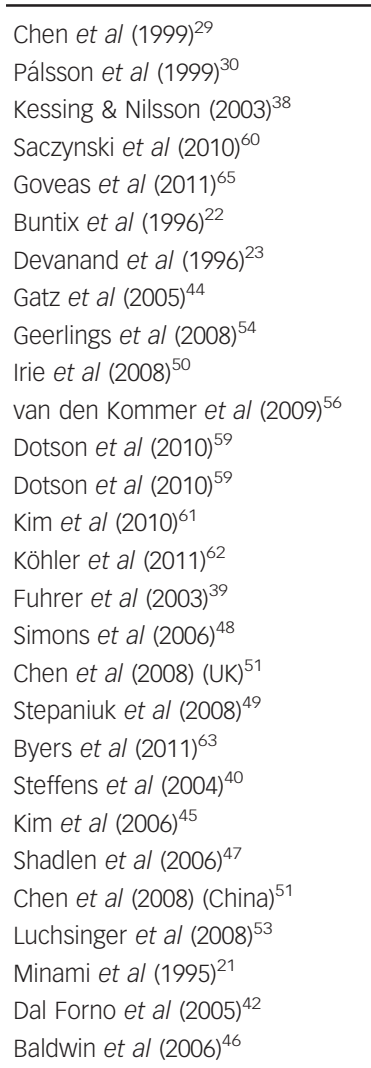

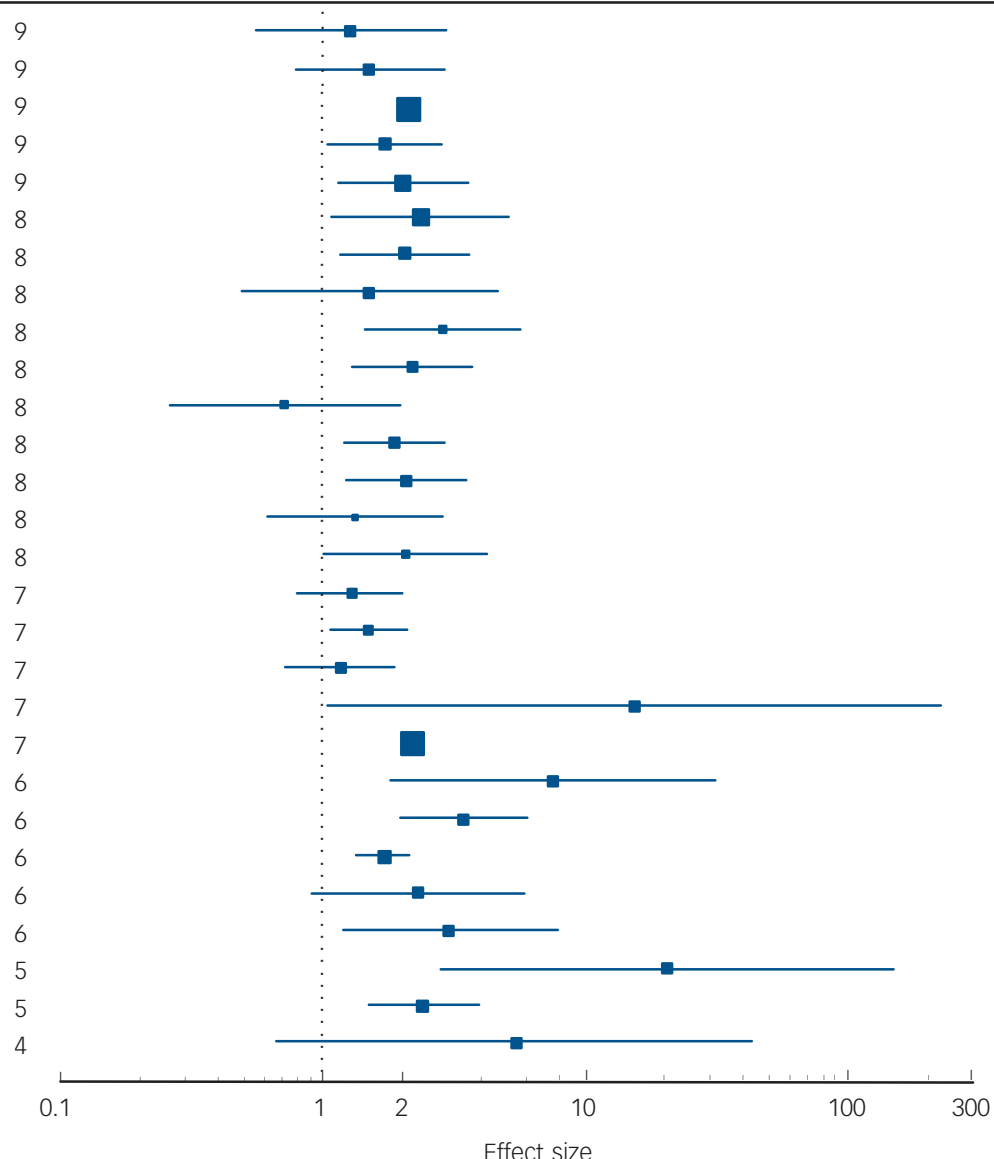

RR: 1.27 (0.55-2.93)

OR: $1.50(0.80-2.90)$

HR: 2.13 (2.00-2.26)

HR: 1.72 (1.04-2.84)

HR: $2.03(1.15-3.60)^{\mathrm{e}}$

OR: 2.38 (1.08-5.06)

RR: 2.05 (1.16-3.62)

OR: $1.50(0.49-4.63)^{\dagger}$

HR: 2.86 (1.45-5.63)

HR: 2.20 (1.30-3.70)

RR: 0.71 (0.26-1.97)

HR: $1.87(1.21-2.88)^{a, c}$

HR: $2.08(1.23-3.52)^{\mathrm{a}, \mathrm{d}}$

OR: $1.33(0.62-2.84)$

OR: 2.06 (1.01-4.22)

OR: 1.30 (0.80-2.00)

HR: 1.50 (1.07-2.10)

RR: 1.17 (0.72-1.88)

OR: 5.20 (1.04-223.22)

HR: 2.18 (2.08-2.28)

RR: $7.50(1.82-31.30)^{b}$

OR: 3.43 (1.98-5.94)

OR: $1.72(1.37-2.15)^{\text {b }}$

RR: 2.30 (0.90-5.88)

HR: 3.00 (1.20-7.80)

OR: 20.64 (2.84-150.20)

RR: 2.41 (1.50-3.87)

RR: $5.36(0.67-43.10)^{b}$

Effect size

Fig. 3 Forest plot of cohort studies that evaluated depression as a risk factor for dementia.

Studies are organised from high quality (top) to lower quality (bottom). ES, effect size; RR, relative risk; OR, odds ratio. a. Samples may overlap. b. Crude odds ratio calculated from the results. c. Risk estimate for one depressive episode. d. Risk estimate for $\geqslant 2$ depressive episodes. e. Risk estimate for history of depression and depression at baseline. f. Risk estimate for late-life depression was also provided: OR $2.75(1.04-7.24)$

irrespective of whether dementia in general was considered or depression onset was limited to 4 or more years before Alzheimer's disease was diagnosed. Both these studies had a prospective design with a long follow-up period and both evaluated depression using the Center for Epidemiologic Studies Depression Scale (CES-D). ${ }^{66}$

However, in another cohort study, ${ }^{48}$ only women with depression had a significantly higher risk for developing dementia $(\mathrm{OR}=1.69,95 \%$ CI $1.06-2.69 v . \mathrm{OR}=1.26,95 \%$ CI $0.70-2.08)$ when more depressed participants (higher tertile) were compared with less depressed participants (lower tertile). Furthermore, in the Women's Health Initiative Memory study, ${ }^{65}$ although depression at baseline was not associated with a higher risk of developing dementia, women with a history of depressive disorder had a higher risk of being diagnosed with probable dementia during the follow-up. In another study, depression and the APOE $\varepsilon 4$ allele were shown to interact and increase the risk for developing dementia $(\mathrm{OR}=5.85,95 \%$ CI $1.77-19.38) .{ }^{61}$ Again, this was statistically significant in men but not in women.

\section{Risk of dementia and interaction between depression and APOE $\varepsilon 4$}

Some studies evaluated the interaction between depression and APOE status. They found that, irrespective of APOE status, the risk of developing dementia is increased in patients with a history of depression. ${ }^{24,50,52,53,60}$ When present together, depression and the $A P O E$ \&4 allele interacted to greatly increase the risk of developing dementia. ${ }^{24,50,57,60}$ Interestingly, this was shown even when depression was not an independent risk factor for dementia. ${ }^{61}$ Other studies seem to contradict these results. Devanand $e a^{23}$ found that when controlling for APOE $\varepsilon 4$ status, depression was still associated with higher risk of dementia, but this association was no longer statistically significant (relative risk $(\mathrm{RR})=2.82,95 \% \mathrm{CI}, 0.93-8.6, P=0.07)$. The data on APOE $\varepsilon 4$ status was only available for less than a third of the sample. Also in disagreement with the previously cited studies, Lenoir et al ${ }^{64}$ did not find an increased risk in APOE $\varepsilon 4$ carriers with depression at baseline.

\section{Bipolar disorder as a risk factor for dementia}

Bipolar disorder has also been evaluated as a putative risk for dementia, ${ }^{19,26,27,38,41}$ albeit much less so than depression (online Table DS5). Risk estimates are higher here than in most studies evaluating depression. Three of these studies ${ }^{27,38,41}$ reported results that achieved statistical significance. In one of these studies, people with one episode of bipolar disorder had a higher risk of developing dementia than individuals with one episode of unipolar depressive disorder (Fig. 6). ${ }^{41}$ In this same study, using as a reference patients with only one episode of bipolar disorder, the risk was higher only for those with four episodes or more. However, this result did not achieve statistical significance. 
Chen et al (1999) 29

Geerlings et al (2000) ${ }^{31}$

Becker et al (2009) ${ }^{55}$

Saczynski et al (2010)

Lenoir et al (2011) ${ }^{64}$

Devanand et al (1996) ${ }^{23}$

Lindsay et al (2002) ${ }^{34}$

Geerlings et al $(2008)^{54}$

Irie et al (2008) $)^{50}$

Jungwirth et al (2009) ${ }^{57}$

Köhler et al (2011) $)^{62}$

Wilson et al (2002) ${ }^{35}$

Andersen et al (2005) ${ }^{43}$

Fischer et al (2008) ${ }^{52}$

Dal Forno et al (2005) ${ }^{42}$

Lenoir et al (2011) $)^{64}$

Irie et al (2008) ${ }^{50}$

Köhler et al (2011) $)^{62}$

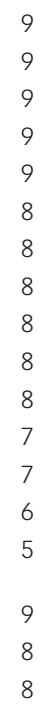

Alzheimer's disease

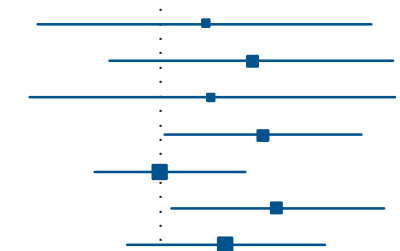

RR: $1.28(0.51-3.2)$

OR: $1.67(0.76-3.63)$

HR: $1.33(0.49-3.65)$

HR: $1.76(1.03-3.01)$

HR: $1.00(0.70-1.60)$

RR: $1.91(1.07-3.42)$

OR: $1.44(0.84-2.48)$

HR: $2.97(1.33-6.61)$

HR: $2.90(1.40-5.90)$

OR: $2.70(1.28-5.70)$

OR: $1.81(0.78-4.23)$

RR: $1.19(1.07-1.32)$

OR: $1.60(0.90-2.70)$

OR: 2.09 (1.25-3.48)

RR: 2.92 (1.70-5.02)

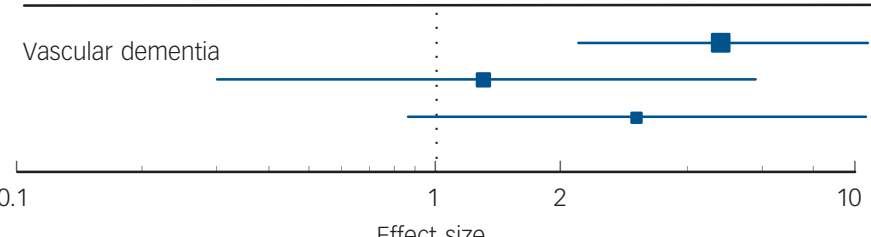

RR: $4.80(2.20-10.70)$

HR: $1.30(0.30-5.80)$

OR: 3.03 (0.86-10.64)

Effect size

Fig. 4 Forest plot of cohort studies that evaluated depression as a risk factor for Alzheimer's disease or vascular dementia.

Studies are organised from high quality (top) to lower quality (bottom). ES, effect size; RR, relative risk; OR, odds ratio; HR, hazard ratio.

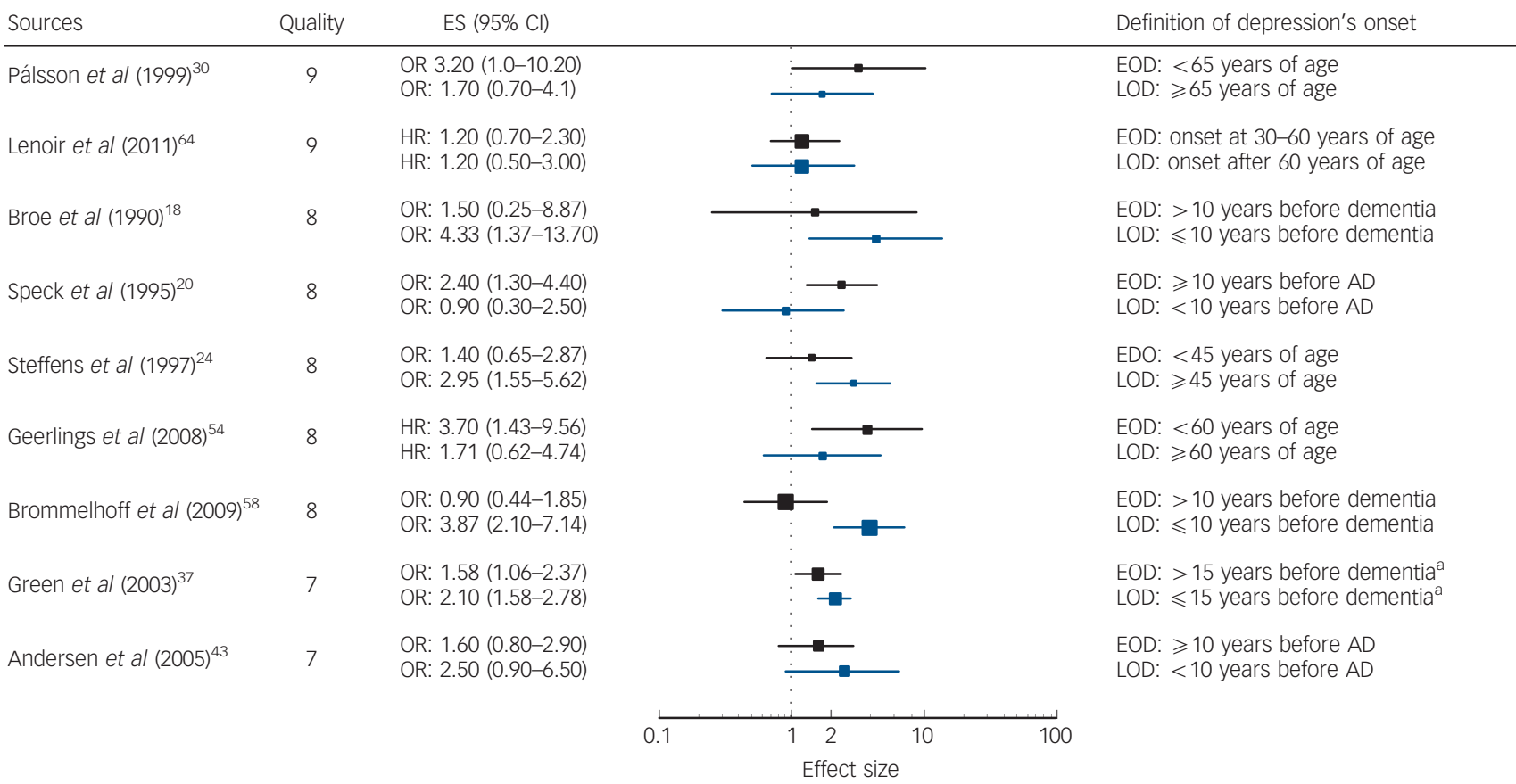

Fig. 5 Forest plot of studies that evaluated risk of dementia associated with both early- and late-onset depression.

Studies are organised from high quality (top) to lower quality (bottom). ES, effect size; OR, odds ratio; HR, hazard ratio; EOD, early-Onset depression; LOD, late-onset depression;

$A D$, Alzheimer's disease.

a. This study evaluated the risk associated with LOD and EOD using different cut-offs for the interval in years between depression onset and Alzheimer's disease onset ( 1 to 25 years). The ORs for the 15 year cut-off is shown here.

\section{Discussion}

In the present review we address the evidence for affective disorders as risk factors for dementia. Current research has been insufficient to fully clarify the links between depression or bipolar disorder and the later development of dementia. However, the data reviewed here may add to our understanding of some issues that have been raised.

\section{Is depression a prodrome or a risk factor for dementia?}

The notion that depression may be a prodrome for dementia has been put forward in a number of publications. ${ }^{29,67,68}$ For example, depression increases the probability of conversion from mild cognitive impairment to dementia. ${ }^{69}$ This has been recently supported by research with peripheral biomarkers. Elderly patients with depression but without cardiovascular disorders exhibit a similar profile to that reported in patients with Alzheimer's 


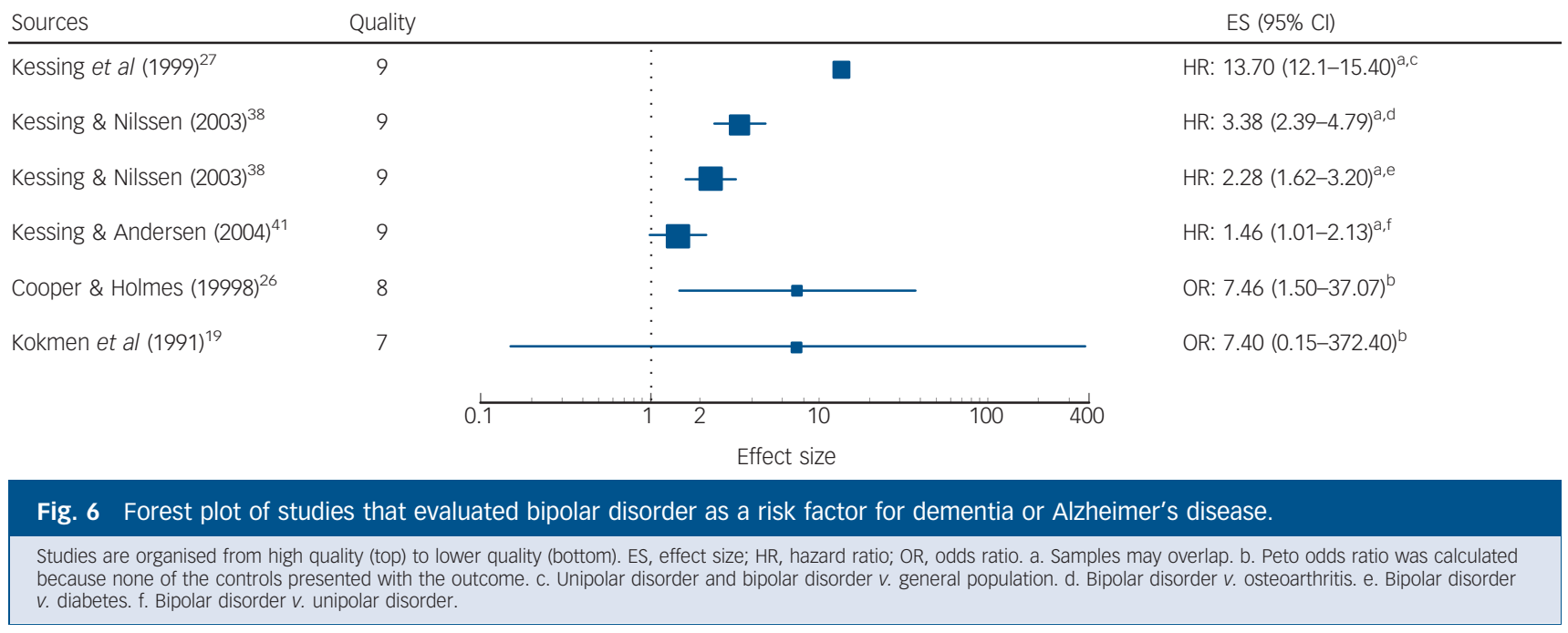

disease: ${ }^{70}$ a decreased plasma concentration of amyloid $(\mathrm{A} \beta)$ peptide $A \beta 42$ with a corresponding increase in the $A \beta 40 / A \beta 42$ ratio. ${ }^{71}$

However, several of the studies included in our review show that the risk of developing dementia is not limited to late-life depression..$^{20,30,37,54}$ In fact, a previous meta-analysis ${ }^{10}$ found that the interval between diagnosis of depression and Alzheimer's disease was positively correlated with an increased risk of developing Alzheimer's disease. Furthermore, many of the prospective studies presented in our review controlled for cognitive scores at baseline and in several of them depression was still found to be significantly associated with a higher risk of developing dementia. ${ }^{23,31,39,54}$

It has also been argued that depression is linked with a higher risk of developing dementia because it is a frequent reaction to the emotional losses associated with cognitive decline. In this regard, Wilson et $a l^{72}$ re-analysed the data of a cohort used to study depressive symptoms and the risk of developing mild cognitive impairment or Alzheimer's disease and could not find an increase in depressive symptoms during the preclinical phase of Alzheimer's disease. This suggests that the association between depressive symptoms and the latter development of Alzheimer's disease is not due to reactive issues.

No unequivocal evidence connects late-onset depression with Alzheimer's disease-like pathology. Therefore, although Alzheimer's disease pathology can start to build up very early in life, ${ }^{73}$ it seems far-fetched to claim that early-life depression is already a manifestation of these neuropathological changes. Furthermore, even in the presence of increased Alzheimer's disease pathological changes in elderly patients with depression, other explanations besides the prodrome theory are valid. For example Rapp et $a l^{74}$ found that people with Alzheimer's disease and a history of depression showed higher plaque and tangle formation within the hippocampus, as compared with those with Alzheimer's disease without a lifetime history of depression. Thus, it can be argued that depression may build on Alzheimer's disease pathology, inducing higher lesion burden. Given these contradictory results, it seems that the risk of developing dementia associated with depressive disorders is not exclusively explained by the prodrome hypothesis.

Contrary to late-life depression, the association between earlylife depression and dementia risk is more difficult to study. It poses greater methodological challenges such as recall bias, retrospective definition of cases or the necessity for a longer follow-up period. In a recent review, Byers \& Yaffe ${ }^{12}$ concluded that early-life depression is a consistent risk factor for dementia but that the results regarding late-life depression were conflicting. Our review does not completely support this perspective. For instance, the results from studies that evaluated both early- and late-life depression as risk factors for dementia are not consistent (Fig. 5). This may be explained by methodological differences, especially divergences with regard to the definition of age at onset of late-onset depression. We cannot exclude the possibility that both early- and late-onset depression are related to an increased risk of developing dementia.

\section{Is there a gender gap?}

The results of studies evaluating both genders independently were surprising and raise important research questions. If depression is a risk factor for males and not females, then causal pathways that offer no logical explanation for observed gender differences should be discarded. ${ }^{39}$ On the other hand, it is known that women tend to recognise non-specific feelings of distress as an emotional problem more promptly than men. Thus, males in these studies may in fact be a group of men with depressive symptoms severe enough to be self-reported. ${ }^{42}$ This may lead to a dilution of the risk in women and an overestimation in men, since, as previously discussed, the risk increases with increased severity of depression at baseline. ${ }^{35,44,50}$ Nonetheless, results supporting a gender gap were not consistent. Furthermore, most studies reported that depression was an independent risk factor for dementia while adjusting for gender. ${ }^{21,23,44,48,53,54,60,62,64}$ It may be that studies that found differences between men and women were the only ones to report the results separately for both genders. In fact, the authors of a previous systematic review on depression and risk of Alzheimer's disease reported that the risk for each gender was only shown separately in those studies that found differences in risk between men and women. ${ }^{10}$

\section{Is bipolar disorder a risk factor for dementia?}

Most of the studies included in this review did not address bipolar disorder as a potential risk factor for dementia. On the other hand, the large majority of studies that considered depression were poorly controlled for bipolar disorder. Thus, some individuals with bipolar depression might have been included in the analyses, probably more so in studies considering early-onset depression. Although the depressive episodes are generally predominant in relation to the mania/hypomania episodes in bipolar disorder, ${ }^{75}$ 
the disorder itself is substantially different from unipolar disorder and bipolar depression has different characteristics when compared with unipolar depression. ${ }^{76}$ Thus, one cannot extrapolate from the data regarding depression to encompass bipolar disorder.

Nevertheless, almost all studies that analysed bipolar disorder as a risk factor for dementia consistently found a higher risk in these patients. ${ }^{19,26,27,38,41}$ Most of these studies used a methodology based on case registers ${ }^{19,26,27,38,41}$ and 2 studies compared individuals with bipolar depression either to people with somatic disorders or to those with other psychiatric disorders. ${ }^{38,41}$ This may occasionally lead to bias. For example, people with osteoarthritis are usually medicated with antiinflammatory drugs, found to be a protective factor for dementia in several studies. ${ }^{77}$ Therefore, this may result in an overestimation of the risk associated with bipolar disorder. However, an increased hazard ratio when comparing individuals with bipolar disorder to people with diabetes is a robust finding, since diabetes is a well-documented risk factor for Alzheimer's disease and vascular dementia. ${ }^{78}$

Another source of bias is the fact that people with bipolar disorder are known to the psychiatric services and this may lead to an increased probability of being admitted throughout one's lifespan, and eventually being diagnosed with dementia in advancing years. However, the opposite could also be argued. It is common in clinical practice to delay a diagnosis of dementia in people with a psychiatric history because behavioural changes and cognitive impairments are wrongly attributed to the psychiatric disorder or to psychopharmacological treatments.

\section{Pathophysiological mechanisms linking depression to the development of dementia}

Two major hypotheses have been put forward to explain the association of late-onset depression with a greater risk of developing dementia: (a) depression is associated with the development of dementia because it is a reaction to an early stage of the cognitive decline; and (b) depression is a prodrome of dementia. Both hypotheses do not concern depression as a causal determinant for dementia. However, these two hypotheses do not fully explain the data presented above, because a higher risk has been described even for patients who had depression several years before dementia's onset. ${ }^{20,30,37,54}$ Thus, a complex interaction of determinants seems more probable than a single and simple aetiological mechanism.

It may be that depression and dementia share common aetiological factors. ${ }^{11}$ For example, inflammation, vascular changes and vascular risk factors have been implicated in both disorders. In this regard, recent studies have controlled for vascular risk factors and vascular disease when evaluating the risk of developing Alzheimer's disease in patients with depression $^{53,60,62-65}$ (Table DS4). Nevertheless, in all of these studies, depression still remained an independent risk factor for Alzheimer's disease. The results of some of the studies reviewed suggest yet another mechanism by reporting a synergistic effect between depression and the APOE $\varepsilon 4$ allele. . $^{4,50,57,60}$

An additional hypothesis is that affective disorders lower the threshold for the clinical manifestation of dementia. The results of this review tend to support this, demonstrating a non-specific increase in the risk of developing dementia (Alzheimer's disease, vascular dementia and dementia in general). Glucocorticoid neurotoxicity may be a plausible underlying mechanism, ${ }^{79-81}$ although longitudinal data derived from human studies is still scarce. $^{54,82}$ Psychosocial factors such as disrupted social interactions and lower lifetime achievements of people with affective disorders, by leading to a lower cognitive reserve, may also decrease the threshold for dementia. However, these remain to be explored. Other mechanisms have been proposed to explain this link but an extensive discussion of pathophysiological mechanisms is outside the scope of this paper (for a comprehensive review see Duman ${ }^{83}$ ).

\section{Methodological issues and limitations}

We used a wide range of search terms together with broadly defined criteria in order to avoid missing important evidence. This strategy enabled us to include a large number of studies. However, these studies were very heterogenous (for example different diagnosis criteria for dementia, different types of dementia, distinct methods for diagnosing depression and differences regarding the onset of depression). Because of this, we decided not to do a meta-analysis and, thus, we were not able to provide summary estimates of the risk of developing dementia in individuals with a history of affective disorders. Instead, we present a wider view of this topic, one that would have been lost if stricter and more homogenised criteria were applied. Nevertheless, to make the strength of the evidence easier to assess, we provided a broad assessment of the quality of each study and comprehensive extraction of data.

When analysing the list of studies retrieved, one can see that the earliest studies were mainly case-control studies in contrast to the most recent ones, which were predominantly cohort studies. The initial case-control studies struggled with recall bias and/or selection bias due to the need to establish retrospectively a diagnosis of depression. ${ }^{15-20,24-26,28,32,33,36,37}$ Generally, the design of these studies relied on proxy informants or clinical records for depression diagnosis, since the patients had dementia. The major problem with using a proxy informant as the only source of information is that it is very difficult to have a reliable diagnosis based on current diagnostic criteria. Similarly, relying exclusively on clinical records could lead to selection bias. For example, people with a depressive illness have a greater probability of being referred to mental health services and thus an increased probability of being identified as having dementia and being included in the group of cases. In spite of this, good agreement has been found between case-control and cohort studies. Jorm ${ }^{11}$ reported in his meta-analysis a summary risk estimate of 2.01 (95\% CI 1.16-3.5) for the case-control studies and 1.87 (1.093.2) for cohort studies. Similar findings were also reported by Ownby et $a l^{10}$ regarding depression and the risk of developing Alzheimer's disease, with an odds ratio of 2.03 (95\% CI $1.73-$ 2.38) for case-control studies and 1.90 (95\% CI 1.55-2.33) for cohort studies with a combined result of 2.02 (95\% CI 1.80-2.26).

Most epidemiological studies reviewed in this paper, as well as previous meta-analyses, showed an increased risk for dementia in individuals with affective disorders. However, publication bias has to be considered (i.e. a lower probability of negative results being published). In fact, in a previous meta-analysis ${ }^{10}$ the funnel plot analysis did indicate that such bias existed in this type of publication. Even so, when the odds ratios were corrected for potential publication bias, there were no significant changes in the risk estimates.

\section{Clinical implications}

It was believed that in elderly people with so-called depressive pseudodementia, cognition and function could be entirely restored to normal if depression was treated. However, this concept has been progressively abandoned since cognitive dysfunction may not be totally reversed by antidepressant 
treatment and more than $40 \%$ of people with pseudodementia eventually develop dementia. ${ }^{84,85}$ Our results support a link between affective disorders and the later development of dementia, especially in men ${ }^{39,42,61}$ and in people with recurrent depressive disorder. ${ }^{41,59}$ Therefore cognitive dysfunction in such patients should not be disregarded as a minor phenomenon. Clinical monitoring of cognition may be warranted, for at least a subset of patients.

\section{Future research}

Previous meta-analyses had not explored some important characteristics that may help in the understanding of the link between depression and dementia. Throughout the present review it has become apparent that the number of disease episodes, severity of depressive symptoms and patient gender are important modulators of the risk of developing dementia. This can help to guide basic research focused on understanding the mechanisms behind this link. Also, the data reviewed suggests that both hypotheses of affective disorders being a prodrome and a risk factor may be consistent. In fact, these hypotheses are not mutually exclusive and in one instance they were even supported by data from the same study. ${ }^{37}$ This should be considered when delineating future research, because both early- and late-onset depression may increase the risk for developing dementia through different mechanisms.

Although the results regarding bipolar disorder are very interesting, the available evidence is still limited. Exploring bipolar disorder as a risk factor for dementia requires more complex methodologies than the ones used for depression. For example, given the lower prevalence of bipolar disorder, a larger sample size is needed. It is also important to control for different types of affective episodes, so that the weight of depressive and manic episodes can be properly evaluated. Similarly, care must be taken to control for treatment because mood stabilisers may be protective factors for dementia in people with bipolar disorder. ${ }^{86-88}$ Thus, there is a need to refine methods in order to clarify this issue.

Given all the possible mechanisms that have been put forward to explain why depression leads to an increased risk of developing dementia, a multifactorial model seems almost inevitable. In fact, Butters et $a l^{89}$ have proposed a multiple pathways model where cerebrovascular disease and glucocorticoid neurotoxicity associated with depression lower the brain reserve and interact with Alzheimer's disease neuropathology, thereby leading to the clinical manifestations of Alzheimer's disease. However, such models are still much more based on associative data then on evidence of causality. Thus, they must be seen more as a 'road map' for future epidemiological and experimental studies, and not as the actual explanation of the link between affective disorders and higher risk of developing dementia. Understanding this link could lead to a better comprehension of the pathophysiology of both depression and Alzheimer's disease, two of the most disabling diseases affecting humankind.

\footnotetext{
Joaquim da Silva, MD, Department of Mental Health and CEDOC, Faculdade de Ciências Médicas, Universidade Nova de Lisboa and Champalimaud Neuroscience Programme, Champalimaud Center for the Unknown, Lisbon, Portugal; Manuel Goncalves-Pereira, MD, PhD, Miguel Xavier MD, PhD, Department of Mental Health and CEDOC, Faculdade de Ciências Médicas, Universidade Nova de Lisbal Health and CEDOC, Faculdade de Ciê. Lisbon, Portugal; Elizabeta B. Mukaetova-Ladinska, MD, MRCPsych, PhD, Institute for Ageing and Health, Campus for Ageing and Vitality, Newcastle University, Newcastle upon Tyne, UK

Correspondence: Joaquim da Silva, Department of Mental Health and CEDOC Faculdade de Ciências Médicas, Universidade Nova de Lisboa, FCM, UNL, Campo Mártires da Pátria, 130, 1169-056 Lisbon, Portugal. Email:

jalvesdasilva@fcm.unl.pt

First received 10 Nov 2011, final revision 27 May 2012, accepted 18 oct 2012
}

\section{Acknowledgements}

We thank Miss Maxine Berwick for secretarial support and editing the manuscript.

\section{References}

1 van der Flier WM, Scheltens P. Epidemiology and risk factors of dementia. J Neurol Neurosurg Psychiatry 2005; 76 (suppl 5): v2-7.

2 Kalaria RN, Maestre GE, Arizaga R, Friedland RP, Galasko D, Hall K, et al. Alzheimer's disease and vascular dementia in developing countries: prevalence, management, and risk factors. Lancet Neurol 2008; 7: 812-26.

3 Ferri $\mathrm{CP}$, Prince $\mathrm{M}$, Brayne $\mathrm{C}$, Brodaty $\mathrm{H}$, Fratiglioni L, Ganguli $\mathrm{M}$, et al. Global prevalence of dementia: a delphi consensus study. Lancet 2005; 366 2112-7.

4 Paelecke-Habermann Y, Pohl J, Leplow B. Attention and executive functions in remitted major depression patients. J Affect Disord 2005; 89: 125-35.

5 Robinson L, Thompson JM, Gallagher P, Goswami U, Young AH, Ferrier IN, et al. A meta-analysis of cognitive deficits in euthymic patients with bipolar disorder. J Affect Disord 2006; 93: 105-15.

6 Sheline YI, Wang PW, Gado MH, Csernansky JG, Vannier MW. Hippocampal atrophy in recurrent major depression. Proc Natl Acad Sci U S A 1996; 93: 3908-13.

7 Sheline YI, Sanghavi M, Mintun MA, Gado MH. Depression duration but not age predicts hippocampal volume loss in medically healthy women with recurrent major depression. J Neurosci 1999; 19: 5034-43.

8 Savitz J, Drevets WC. Bipolar and major depressive disorder: neuroimaging the developmental-degenerative divide. Neurosci Biobehav Rev 2009; 33: 699-771.

9 Kraepelin E. Lectures on Clinical Psychiatry. Bailliere, Tindall and Cox, 1913.

10 Ownby RL, Crocco E, Acevedo A, John V, Loewenstein D. Depression and risk for Alzheimer disease: systematic review, meta-analysis, and metaregression analysis. Arch Gen Psychiatry 2006; 63: 530-8.

11 Jorm AF. History of depression as a risk factor for dementia: an updated review. Aust N Z J Psychiatry 2001; 35: 776-81.

12 Byers AL, Yaffe K. Depression and risk of developing dementia. Nat Rev Neurol 2011; 7: 323-31.

13 Haynes RB, Wilczynski N, McKibbon KA, Walker CJ, Sinclair JC. Developing optimal search strategies for detecting clinically sound studies in MEDLINE. J Am Med Inform Assoc 1994; 1: 447-58.

14 Wells GA, Shea B, O'Connell D, Peterson J, welch V, Losos M, et al. The Newcastle-Ottawa Scale (NOS) for Assessing the Quality of Nonrandomised Studies in Meta-Analyses. Ottawa Hospital Research Institute, 2011 (http:// www.ohri.ca/programs/clinical_epidemiology/oxford.asp).

15 French LR, Schuman LM, Mortimer JA, Hutton JT, Boatman RA, Christians B. A case-control study of dementia of the Alzheimer type. Am J Epidemiol 1985; 121: 414-21.

16 Agbayewa MO. Earlier psychiatric morbidity in patients with Alzheimer's disease. J Am Geriatr SOC 1986; 34: 561-4.

17 Shalat SL, Seltzer B, Pidcock C, Baker EL. Risk factors for Alzheimer's disease: a case-control study. Neurology 1987; 37: 1630-3.

18 Broe GA, Henderson AS, Creasey $H$, McCusker E, Korten AE, Jorm AF, et al. A case-control study of Alzheimer's disease in australia. Neurology 1990; 40: 1698-707.

19 Kokmen E, Beard CM, Chandra V, Offord KP. Clinical risk factors for Alzheimer's disease: a population-based case-control study. Neurology 1991; 41: 1393-7.

20 speck CE, Kukull WA, Brenner DE, Bowen JD, McCormick WC, Teri L, et al. History of depression as a risk factor for Alzheimer's disease. Epidemiology 1995; 6: 366-9.

21 Minami Y, Tsuji I, Fukao A, Hisamichi S, Asano H, Sato M, et al. Physical status and dementia risk: a three-year prospective study in urban Japan. Int J Soc Psychiatry 1995; 41: 47-54.

22 Buntinx F, Kester A, Bergers J, Knottnerus JA. Is depression in elderly people followed by dementia? A retrospective cohort study based in general practice. Age Ageing 1996; 25: 231-3.

23 Devanand DP, Sano M, Tang MX, Taylor S, Gurland BJ, Wilder D, et al. Depressed mood and the incidence of Alzheimer's disease in the elderly living in the community. Arch Gen Psychiatry 1996; 53: 175-82.

24 Steffens DC, Plassman BL, Helms MJ, Welsh-Bohmer KA, Saunders AM, Breitner JC. A twin study of late-onset depression and apolipoprotein $\mathrm{E}$ epsilon 4 as risk factors for Alzheimer's disease. Biol Psychiatry 1997; 41: 851-6. 
25 Tsolaki M, Fountoulakis K, Chantzi E, Kazis A. Risk factors for clinically diagnosed Alzheimer's disease: a case-control study of a Greek population. Int Psychogeriatr 1997; 9: 327-41.

26 Cooper B, Holmes C. Previous psychiatric history as a risk factor for late-life dementia: a population-based case-control study. Age Ageing 1998; 27: 181-8.

27 Kessing LV, Olsen EW, Mortensen PB, Andersen PK. Dementia in affective disorder: a case-register study. Acta Psychiatr Scand 1999; 100: 176-85.

28 Wetherell JL, Gatz M, Johansson B, Pedersen NL. History of depression and other psychiatric illness as risk factors for Alzheimer disease in a twin sample. Alzheimer Dis Assoc Disord 1999; 13: 47-52.

29 Chen P, Ganguli M, Mulsant BH, DeKosky ST. The temporal relationship between depressive symptoms and dementia: a community-based prospective study. Arch Gen Psychiatry 1999; 56: 261-6.

30 Pálsson S, Aevarsson O, Skoog I. Depression, cerebral atrophy, cognitive performance and incidence of dementia. Population study of 85 -year-olds. Br J Psychiatry 1999; 174: 249-53.

31 Geerlings MI, Schoevers RA, Beekman ATF, Jonker C, Deeg DJH, Schmand B, et al. Depression and risk of cognitive decline and Alzheimer's disease: results of two prospective community-based studies in The Netherlands. Br J Psychiatry 2000; 176: 568-75.

32 Zalsman G, Aizenberg D, Sigler M, Nahshony E, Karp L, Weizman A. Increased risk for dementia in elderly psychiatric inpatients with late-onset major depression. J Nerv Ment Dis 2000; 188: 242-3.

33 Hébert R, Lindsay J, Verreault R, Rockwood K, Hill G, Dubois MF. Vascular dementia: incidence and risk factors in the Canadian study of health and aging. Stroke 2000; 31: 1487-93.

34 Lindsay J, Laurin D, Verreault R, Hébert R, Helliwell B, Hill GB, et al. Risk factors for Alzheimer's disease: a prospective analysis from the Canadian study of health and aging. Am J Epidemiol 2002; 156: 445-53.

35 Wilson RS, Barnes LL, Mendes DL, Aggarwal NT, Schneider JS, Bach J, et al. Depressive symptoms, cognitive decline, and risk of Alzheimer's disease in older persons. Neurology 2002; 59: 364-70.

36 Zubenko GS, Zubenko WN, MCPherson S, Spoor E, Marin DB, Farlow MR, et al. A collaborative study of the emergence and clinical features of the major depressive syndrome of Alzheimer's disease. Am J Psychiatry 2003; 160: 857-66

37 Green RC, Cupples LA, Kurz A, Auerbach S, Go R, Sadovnick D, et al. Depression as a risk factor for Alzheimer disease: the MIRAGE study. Arch Neurol 2003; 60: 753-9.

38 Kessing LV, Nilsson FM. Increased risk of developing dementia in patients with major affective disorders compared to patients with other medical illnesses. J Affect Disord 2003; 73: 261-9.

39 Fuhrer R, Dufouil C, Dartigues JF. Exploring sex differences in the relationship between depressive symptoms and dementia incidence: prospective results from the PAQUID study. J Am Geriatr Soc 2003; 51: 1055-63.

40 Steffens DC, Welsh-Bohmer KA, Burke JR, Plassman BL, Beyer JL, Gersing KR, et al. Methodology and preliminary results from the neurocognitive outcomes of depression in the elderly study. J Geriatr Psychiatry Neurol 2004; 17: 202-11.

41 Kessing LV, Andersen PK. Does the risk of developing dementia increase with the number of episodes in patients with depressive disorder and in patients with bipolar disorder? J Neurol Neurosurg Psychiatry 2004; 75: 1662-6.

42 Dal Forno G, Palermo MT, Donohue JE, Karagiozis $H$, Zonderman AB, Kawas $\mathrm{CH}$. Depressive symptoms, sex, and risk for Alzheimer's disease. Ann Neurol 2005; 57: 381-7.

43 Andersen K, Lolk A, Kragh-Sørensen P, Petersen NE, Green A. Depression and the risk of Alzheimer disease. Epidemiology 2005; 16: 233-8.

44 Gatz JL, Tyas SL, St John P, Montgomery P. Do depressive symptoms predict Alzheimer's disease and dementia? J Gerontol A Biol Sci Med Sci 2005; 60 744-7.

45 Kim JM, Stewart R, Kim SW, Yang SJ, Shin IS, Yoon JS. A prospective study of changes in subjective memory complaints and onset of dementia in South Korea. Am J Geriatr Psychiatry 2006; 14: 949-56.

46 Baldwin RC, Gallagley A, Gourlay M, Jackson A, Burns A. Prognosis of late life depression: a three-year cohort study of outcome and potential predictors. Int J Geriatr Psychiatry 2006; 21: 57-63.

47 Shadlen MF, Siscovick D, Fitzpatrick AL, Dulberg C, Kuller LH, Jackson S. Education, cognitive test scores, and black-white differences in dementia risk. J Am Geriatr SOC 2006; 54: 898-905.

48 Simons LA, Simons J, McCallum J, Friedlander Y. Lifestyle factors and risk of dementia: Dubbo study of the elderly. Med J Aust 2006; 184: 68-70.

49 Stepaniuk J, Ritchie LJ, Tuokko H. Neuropsychiatric impairments as predictors of mild cognitive impairment, dementia, and Alzheimer's disease. Am J Alzheimers Dis Other Demen 2008; 23: 326-33.
50 Irie F, Masaki KH, Petrovitch H, Abbott RD, Ross GW, Taaffe DR, et al Apolipoprotein E epsilon4 allele genotype and the effect of depressive symptoms on the risk of dementia in men: the Honolulu-Asia aging study. Arch Gen Psychiatry 2008; 65: 906-12.

51 Chen R, Hu Z, Wei L, Qin X, Mccracken C, Copeland JR. Severity of depression and risk for subsequent dementia: cohort studies in China and the UK. Br J Psychiatry 2008; 193: 373-7.

52 Fischer $\mathrm{P}$, Zehetmayer $\mathrm{S}$, Jungwirth $\mathrm{S}$, Weissgram $\mathrm{S}$, Krampla W, Hinterberger $\mathrm{M}$, et al. Risk factors for Alzheimer dementia in a community-based birth cohort at the age of 75 years. Dement Geriatr Cogn Disord 2008; 25: 501-7.

53 Luchsinger JA, Honig LS, Tang MX, Devanand DP. Depressive symptoms, vascular risk factors, and Alzheimer's disease. Int J Geriatr Psychiatry 2008; 23: 922-8.

54 Geerlings MI, den Heijer T, Koudstaal PJ, Hofman A, Breteler MMB. History of depression, depressive symptoms, and medial temporal lobe atrophy and the risk of Alzheimer disease. Neurology 2008; 70: 1258-64.

55 Becker JT, Chang YF, Lopez OL, Dew MA, Sweet RA, Barnes D, et al. Depressed mood is not a risk factor for incident dementia in a communitybased cohort. Am J Geriatr Psychiatry 2009; 17: 653-63.

56 van den Kommer TN, Bontempo DE, Comijs HC, Hofer SM, Dik MG, Piccinin $\mathrm{AM}$, et al. Classification models for early identification of persons at risk for dementia in primary care: an evaluation in a sample aged 80 years and older. Dement Geriatr Cogn Disord 2009; 28: 567.

57 Jungwirth S, Zehetmayer S, Bauer P, Weissgram S, Tragl KH, Fischer P. Prediction of Alzheimer dementia with short neuropsychological instruments. J Neural Transm 2009; 116: 1513-21.

58 Brommelhoff JA, Gatz M, Johansson B, McArdle JJ, Fratiglioni L, Pedersen NL. Depression as a risk factor or prodromal feature for dementia? Findings in a population-based sample of swedish twins. Psychol Aging 2009; 24: 373-84.

59 Dotson VM, Beydoun MA, Zonderman AB. Recurrent depressive symptoms and the incidence of dementia and mild cognitive impairment. Neurology 2010; 75: 27-34.

60 Saczynski JS, Beiser A, Seshadri S, Auerbach S, Wolf PA, Au R. Depressive symptoms and risk of dementia: the Framingham Heart Study. Neurology 2010; 75: 35-41.

$61 \mathrm{Kim}$ JM, Kim SY, Bae KY, Kim SW, Shin IS, Yang SJ, et al. Apolipoprotein e4 genotype and depressive symptoms as risk factors for dementia in an older Korean population. Psychiatry Investig 2010; 7: 135-40.

62 Köhler S, van Boxtel M, Jolles J, Verhey F. Depressive symptoms and risk for dementia: a 9-year follow-up of the Maastricht Aging Study. Am J Geriatr Psychiatry 2011; 19: 902-5.

63 Byers AL, Covinsky KE, Barnes DE, Yaffe K. Dysthymia and depression increase risk of dementia and mortality among older veterans. Am J Geriatr Psychiatry 2012; 20: 664-72.

64 Lenoir H, Dufouil C, Auriacombe S, Lacombe JM, Dartigues JF, Ritchie K, et al. Depression history, depressive symptoms, and incident dementia: the $3 \mathrm{C}$ study. J Alzheimers Dis 2011; 26: 27-38.

65 Goveas JS, Espeland MA, Woods NF, Wassertheil-Smoller S, Kotchen JM. Depressive symptoms and incidence of mild cognitive impairment and probable dementia in elderly women: the women's health initiative memory study. J Am Geriatr Soc 2011; 59: 57-66.

66 Radloff LS. The CES-D Scale. Appl Psychol Meas 1977; 1: 385-401.

67 Ganguli M, Du Y, Dodge HH, Ratcliff GG, Chang CC. Depressive symptoms and cognitive decline in late life: a prospective epidemiological study. Arch Gen Psychiatry 2006; 63: 153-60.

68 Schweitzer I, Tuckwell V, O'Brien J, Ames D. Is late onset depression a prodrome to dementia? Int J Geriatr Psychiatry 2002; 17: 997-1005.

69 Panza F, Frisardi V, Capurso C, D'Introno A, Colacicco AM, Imbimbo BP, et al. Late-life depression, mild cognitive impairment, and dementia: possible continuum? Am J Geriatr Psychiatry 2010; 18: 98-116.

70 Yaffe K, Weston A, Graff-Radford NR, Satterfield S, Simonsick EM, Younkin SG, et al. Association of plasma beta-amyloid level and cognitive reserve with subsequent cognitive decline. JAMA 2011; 305: 261-6.

71 Qiu WQ, Sun X, Selkoe DJ, Mwamburi DM, Huang T, Bhadela R, et al. Depression is associated with low plasma abeta42 independently of cardiovascular disease in the homebound elderly. Int J Geriatr Psychiatry 2007; 22: 536-42.

72 Wilson RS, Arnold SE, Beck TL, Bienias JL, Bennett DA. Change in depressive symptoms during the prodromal phase of Alzheimer disease. Arch Gen Psychiatry 2008; 65: 439-45.

73 Ohm TG, Müller H, Braak H, Bohl J. Close-meshed prevalence rates of different stages as a tool to uncover the rate of Alzheimer's disease-related neurofibrillary changes. Neuroscience 1995; 64: 209-17. 
74 Rapp MA, Schnaider-Beeri M, Grossman HT, Sano M, Perl DP, Purohit DP, et al. Increased hippocampal plaques and tangles in patients with Alzheimer disease with a lifetime history of major depression. Arch Gen Psychiatry 2006; 63: 161-7.

75 Judd LL, Akiskal HS. Depressive episodes and symptoms dominate the longitudinal course of bipolar disorder. Curr Psychiatry Rep 2003; 5: 417-8.

76 Bowden CL. A different depression: Clinical distinctions between bipolar and unipolar depression. J Affect Disord 2005; 84: 117-25.

77 Szekely CA, Thorne JE, Zandi PP, Ek M, Messias E, Breitner JC, et al. Nonsteroidal anti-inflammatory drugs for the prevention of Alzheimer's disease: a systematic review. Neuroepidemiology 2004; 23: 159-69.

78 Craft S. The role of metabolic disorders in Alzheimer disease and vascular dementia: two roads converged. Arch Neurol 2009; 66: 300-5.

79 Sapolsky RM, Uno H, Rebert CS, Finch CE. Hippocampal damage associated with prolonged glucocorticoid exposure in primates. J Neurosci 1990; 10 2897-902.

80 Magariños AM, McEwen BS. Stress-induced atrophy of apical dendrites of hippocampal ca3c neurons: involvement of glucocorticoid secretion and excitatory amino acid receptors. Neuroscience 1995; 69: 89-98.

81 Colla M, Kronenberg G, Deuschle M, Meichel K, Hagen T, Bohrer M, et al. Hippocampal volume reduction and hpa-system activity in major depression. J Psychiatr Res 2007; 41: 553-60.
82 O'Brien JT, Lloyd A, McKeith I, Gholkar A, Ferrier N. A longitudinal study of hippocampal volume, cortisol levels, and cognition in older depressed subjects. Am J Psychiatry 2004; 161: 2081-90.

83 Duman RS. Neuronal damage and protection in the pathophysiology and treatment of psychiatric illness: stress and depression. Dialogues Clin Neurosci 2009; 11: 239-55.

84 Alexopoulos GS, Meyers BS, Young RC, Mattis S, Kakuma T. The course of geriatric depression with "reversible dementia": a controlled study. Am J Psychiatry 1993; 150: 1693-9.

85 Kral VA, Emery OB. Long-term follow-up of depressive pseudodementia of the aged. Can J Psychiatry 1989; 34: 445-6.

86 Kessing LV, Sondergard L, Forman JL, Andersen PK. Lithium treatment and risk of dementia. Arch Gen Psychiatry 2008; 65: 1331-5.

87 Kessing LV, Forman JL, Andersen PK. Does lithium protect against dementia? Bipolar Disord 2010; 12: 87-94.

88 Nunes PV, Forlenza OV, Gattaz WF. Lithium and risk for Alzheimer's disease in elderly patients with bipolar disorder. Br J Psychiatry 2007; 190: 359-60.

89 Butters MA, Young JB, Lopez O, Aizenstein HJ, Mulsant BH, Reynolds CF, et al. Pathways linking late-life depression to persistent cognitive impairment and dementia. Dialogues Clin Neurosci 2008; 10: 345-57.

Long shrouded in conceptual and nosological ambiguity, gambling addiction is set to be classified under addictive disorders in DSM-5. Gambling addiction has a general population prevalence of approximately $1 \%$. It cuts across age, gender, class and culture, and has a negative impact on the person's physical and psychological health, finances and family. Despite high comorbidity among those with psychiatric disorders, professionals' limited awareness leads to it going undetected. Simple screening tools aid early detection and brief psychological interventions are effective. Cognitive-behavioural therapies are the treatment of choice and although no drug is licensed for use, opioid antagonists show promise. 